\title{
Hybrid immunoaffinity-mass spectrometric methods for efficient protein biomarker verification in pharmaceutical development
}

\author{
"Hybrid immunoaffinity-mass spectrometry methods ... hold significant importance \\ for the discovery and development of novel protein biomarkers."
}

In the discovery and development of novel protein biomarkers, mass spectrometry (MS) and ligand-binding (LB) assays have traditionally operated at different ends of a common continuum. Liquid chromatography (LC)-MS/MS, with its ability to simultaneously measure thousands of peptides in a single run, has driven the development of proteomics, one of the primary tools used in the search for novel protein biomarkers. LB assays, on the other hand, have been applied to the quantitative analysis of discrete proteins and are the method of choice for clinical diagnostic assays. In simple terms, LC-MS/MS has been primarily used for hypothesis generation, while LB methods have typically been reserved for hypothesis testing. Interestingly, with the trend towards targeted multiprotein analysis (using either MS or LB) growing, the protein biomarker landscape has changed, meaning the roles of these methodologies are far less well defined. As discussed in this article, a positive outcome of this research is the development of hybrid immunoaffinity (IA)-MS methods, which hold significant importance for the discovery and development of novel protein biomarkers.

\section{The protein biomarker pipeline}

It is clear that the immense effort poured into protein biomarker discovery during the past decade has not yielded the results anticipated. In an effort to stimulate more coordinated development efforts towards protein biomarkers, Rifai et al. introduced the notion of the protein biomarker pipeline [1]. The protein biomarker pipeline has four stages: discovery, qualification, verification and validation.

Discovery can occur using either non biased methods, such as bottom-up proteomics or more targeted approaches, such as protein arrays.
Owing to high rates of false discovery, candidate markers must be confirmed in the qualification stage using a more selective secondary method. Western blotting is typically used in cases where antibodies for the identified proteins are available. A more recent alternative is targeted LC-MS/MS using multiple reaction monitoring (MRM) detection to quantify tryptic fragments for proteins of interest. In the latter method, stable isotope-labeled versions of the peptides are employed as internal standards to improve analytical precision [2].

For clinical utility, protein biomarkers must ultimately be detected in an accessible fluid or tissue. The transition from a proximal to an accessible matrix occurs during the verification stage. Unfortunately, LC-MRM-MS methods lack the sensitivity required to interrogate low-abundance protein biomarkers in accessible fluids. A representative example of the current status of LC-MRM-MS can be found in a recent publication by Keshishian and co-workers who quantified six proteins in human plasma with lower limits of quantification (LLOQ) in the range of $1-10 \mathrm{ng} / \mathrm{ml}$, albeit following extensive fractionation [3]. Given the current surplus in biomarker candidates and the need to bridge to clinical ELISA, where assays below $0.1 \mathrm{ng} / \mathrm{ml}$ are frequently needed (i.e., the validation stage), it is not surprising that many people now consider the verification rate limiting to protein biomarker development [4].

\section{Hybrid methods: \\ immunoaffinity-MS}

To permit MS quantification of low-abundance proteins, several investigators have explored hybrid techniques coupling immunoaffinity enrichment with MS detection [5-9]. Lower LLOQs are possible by immunoaffinity

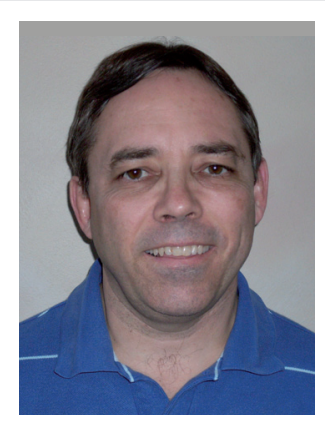

Bradley L Ackermann Senior Research Advisor, Eli Lilly and Company, Lilly Corporate Center, Indianapolis, IN 46285, Mail Drop Code 07I0, USA Tel.: + I 3172764836 Fax: + I 3174336432 E-mail: brad.ackermann@ lilly.com 
(IA)-MS (0.1-1 $\mathrm{ng} / \mathrm{ml}$ in plasma or serum), due to the ability of antibodies to enrich the target protein by as much as 1000-fold [9].

Considerable diversity exists among IA-MS applications. Table I compares two strategies representing the extremes currently encountered in protein biomarker verification. Given the vast number of proteins identified by proteomics, coupled with high rates of false discovery, the multiplexed strategy for targeted proteomics seeks faster methods to interrogate protein biomarker candidates. The primary emphasis of this work is to increase the number of available disease biomarkers for general clinical use [4]. Although IA-MS methods may employ either antiprotein or antipeptide antibodies, the latter option (also known as stable isotope standards with capture by anti-peptide antibody [SISCAPA] [7]) has become popular, owing to its compatability with highly multiplexed analysis. The feasibility of multiplexed analysis is, in part, due to having a universal method for front-end sample preparation (i.e., using the digestion protocols applied in bottom-up proteomics). More complete information about the multiplexing strategy can be found in a recent article by Carr and Anderson [4].

Previously, we published an IA-MS strategy built on a more focused approach that serves as an alternative to the multiplexed method [10] . We have found that this strategy is better suited to the needs of pharmaceutical development. As indicated in Table I, the method relies exclusively on antiprotein antibodies and examines either a single protein or a finite set. The goal of the focused strategy is twofold. First, because

\section{Table 1. Strategies for protein biomarker verification.}

\begin{tabular}{|c|c|c|}
\hline \multirow[t]{2}{*}{ Attribute } & \multicolumn{2}{|c|}{ Strategy } \\
\hline & Multiplexed & Focused \\
\hline Primary emphasis & Disease biomarkers & Pharmacodynamic biomarkers \\
\hline Intended venue & Clinical chemistry & Drug development \\
\hline Goal & $\begin{array}{l}\text { Simultaneous interrogation } \\
\text { of multiple protein } \\
\text { biomarker candidates }\end{array}$ & $\begin{array}{l}\text { Fast interrogation of high- } \\
\text { priority biomarker candidates } \\
\text { and reduced cost for } \\
\text { ELISA development }\end{array}$ \\
\hline $\begin{array}{l}\text { Number of proteins } \\
\text { measured }\end{array}$ & Five to 20 & One to three \\
\hline Antibody target & Protein or tryptic fragment & Protein \\
\hline LLOQ (protein) & $1-10 \mathrm{ng} / \mathrm{ml}$ & $0.1-1 \mathrm{ng} / \mathrm{ml}$ \\
\hline Transition to ELISA & Slow & Fast \\
\hline $\begin{array}{l}\text { Level of method } \\
\text { validation }\end{array}$ & Low to moderate & High \\
\hline
\end{tabular}

only a single capture antibody is required, novel proteins can be evaluated prior to initiating full investment in the costly development of a sandwich ELISA method. Second, IA-MS can further be used to guide ELISA development, ultimately confirming the selectivity of the ELISA method through crossvalidation [11-12].

An important advantage of the focused approach is that it is logistically easier to perform more rigorous method optimization and validation compared with multiplexed methods. Moreover, focused methods tend to have lower LLOQs owing to higher affinity of antiprotein over antipeptide antibodies. Based on published literature, this advantage is currently at least tenfold. Finally, the use of anti-protein antibodies also assists ELISA development as they often are often incorporated into sandwich methods.

While both strategies are important for protein biomarker verification, the focused approach has yet to be fully exploited for pharmaceutical development. In the remainder of this article, issues related to implementation of this approach are more fully explored.

\section{Preferred path for method development}

Many of our initial IA-MS applications were derived from situations where an ELISA method had run into difficulty. Common issues included: concerns about antibody specificity, the lack of a suitable secondary antibody, and matrix effects that limited precision, recovery and/or parallelism. During these investigations, we frequently asked the question, would it not have been better to invest in an IA-MS method in the first place?

The advantage to IA-MS is that protein biomarkers can be evaluated as soon as a single capture antibody is available. Hence, the utility of a candidate marker can be assessed before committing significant resources to create and screen multiple antibodies to identify suitable pairs for sandwich methods. Moreover, it is important to note that focused IA-MS methods not only have the sensitivity needed for clinical biomarker applications, but can be validated according to the expectations of definitive biomarker quantification [13].

At present, two primary obstacles restrict uptake of the proposed IA-MS protein biomarker strategy: access to tools and the inflexibility of current assay development strategies. The first issue refers specifically to 
the cost and availability of mass spectrometers and qualified analysts. The fact that relatively few laboratories or teams have comparable access to MS and LB methods is also a part of this problem.

The second obstacle is less straightforward since it involves matters related to problemsolving methodologies, method ownership and institutional hierarchy. To help understand this situation, Figure I depicts four different paths to the development of a method for a protein biomarker. In each case initial method development begins with either ELISA or MS (indicated as $t_{0}$ ). In path $A$, the method starts and finishes with ELISA (final assay conditions denoted by $t_{f}$ ). This is the path available to many LB groups that do not have access to MS (or lack proficiency). Path B also utilizes a single technology throughout assay development. This is the situation often found in proteomics groups. While several problems can be solved with MS alone, a common pitfall is not recognizing the need for a coordinated transition to LB. Path C is the aforementioned hybrid situation, where MS is used to assist a troubled ELISA method. Although $t_{f}$ in this case is indicated as MS, it should be noted that several iterations may occur. In fact, path $\mathrm{C}$ scenarios often result in an eventual transition back to ELISA once a qualified method is available.

In our view, for the reasons cited earlier, path D represents the most efficient strategy for protein biomarker assay development. This statement must be prefaced with an acknowledgement that no single path represents the best solution in all cases. For instance, IA-MS methods are sometimes unfeasible due to issues of sensitivity and/or selectivity. Throughput may also be a consideration.

\section{Future perspective}

For drug development, the protein biomarker laboratory of the future will have comparable fluency and access to both LB and MS methods. The path towards this vision will be aided by the inevitability that MS technology

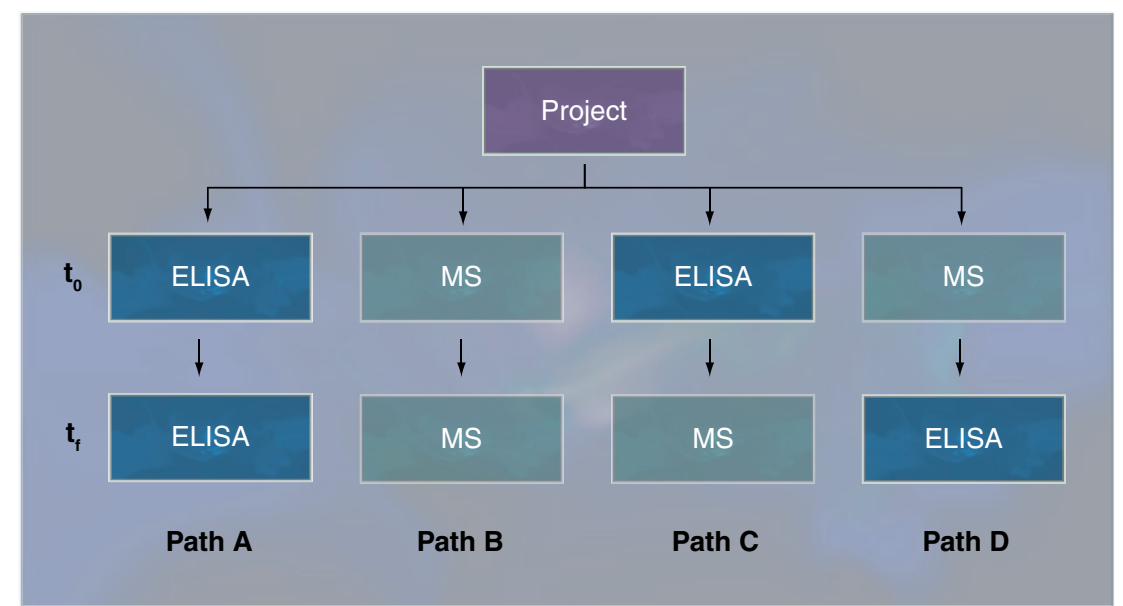

Figure 1. Assay development strategies for protein biomarker methods. MS: Mass spectrometry.

will become more sensitive and affordable. It is also helped by the common-sense awareness that these rapidly evolving technologies return more when working in synergy than as parallel techniques motivated by competition.

Given the reported Human Proteome Organization Antibody Initiative [101], as well as other efforts aimed at increasing the number of available antibodies, greater demand for IAMS in the future is all but certain. Within the context of IA-MS, there will also be a need to better define how fit-for-purpose validation accompanies biomarker methods used in drug development. As this occurs, the selectivity of MS and its ability to track recovery using stable isotope-labeled internal standards will solidify the role of focused IA-MS in the development of novel protein biomarkers.

\section{Financial \& competing interests disclosure}

The author has no relevant affiliations or financial involvement with any organization or entity with a financial interest in or financial conflict with the subject matter or materials discussed in the manuscript. This includes employment, consultancies, honoraria, stock ownership or options, expert testimony, grants or patents received or pending, or royalties.

No writing assistance was utilized in the production of this manuscript.

\section{Bibliography}

1 Rifai N, Gillette MA, Carr SA. Protein biomarker discovery and validation: the long and uncertain path to clinical utility. Nat. Biotechol. 24(8), 971-983 (2006).

2 Gerber SA, Rush J, Stemman O, Kirschner MW, Gygi SP. Absolute quantification of proteins and phosphoproteins from cell lysates by tandem MS. Proc. Natl Acad. Sci. USA 100 (12), 6940-6945 (2003).

3 Keshishian H, Addona T, Burgess M, Kuhn E, Carr SA. Quantitative, multiplexed assays for low abundance proteins in plasma by targeted mass spectrometry and stable isotope dilution. Mol. Cell. Proteomics 6, 2212-2229 (2007).
4 Carr SA, Anderson L. Protein quantitation through targeted mass spectrometry: the way out of biomarker purgatory? Clin. Chem. 54(11), 1749-1752 (2008).

5 Berna M, Ott L, Engle S et al. Quantification of NTproBNP in rat serum using immunoprecipitation and LC/MS/ MS: a biomarker of drug-induced cardiac 


\section{EDITORIAL | Ackermann}

hypertrophy. Anal. Chem. 80(3), 561-566 (2008).

6 Berna MJ, Zhen Y, Watson DE, Hale JE, Ackermann BL. Strategic use of immunoprecipitation and LC/MS/MS for trace-level protein quantification: myosin light chain 1, a biomarker of cardiac necrosis. Anal. Chem. 79(11), 4199-4205 (2007).

7 Anderson NL, Anderson NG, Haines LR et al. Mass spectrometric quantitation of peptides and proteins using Stable Isotope Standards and Capture by Anti-Peptide Antibodies (SISCAPA). J. Proteome Res. 3(2), 235-244 (2004).

8 Whiteaker JR, Zhang H, Zhao L et al. Integrated pipeline for mass spectrometrybased discovery and confirmation of biomarkers demonstrated in a mouse model of breast cancer. J. Proteome Res. 6(10), 3962-3975 (2007).

9 Whiteaker JR, Zhao L, Zhang HY et al. Antibody-based enrichment of peptides on magnetic beads for mass-spectrometry-based quantification of serum biomarkers. Anal. Biochem. 362(1), 44-54 (2007).

10 Ackermann BL, Berna MJ. Coupling immunoaffinity techniques with MS for quantitative analysis of low-abundance protein biomarkers. Expert Rev. Proeteomics 4(2), 175-186 (2007).

11 Oe $\mathrm{T}$, Ackermann BL, Inoue $\mathrm{K}$ et al. Quantitative analysis of amyloid $\beta$ peptides in cerebrospinal fluid of Alzheimer's disease patients by immunoaffinity purification and stable isotope dilution liquid chromatography/ negative electrospray ionization tandem mass spectrometry. Rapid Commun. Mass Spectrom. 20(24), 3723-3735 (2006).

12 Zhen EY, Berna MJ, Jin Z et al. Quantification of heart fatty acid binding protein as a biomarker for drug-induced cardiac and musculoskeletal necroses. Proteomics Clin. Appl. 1, 661-671 (2007).

13 Lee JW, Devanarayan V, Barrett YC et al. Fit-for-purpose method development and validation for successful biomarker measurement. Pharm. Res. 23(2), 312-328 (2006).

\section{- Website}

101 Human Antibody Initiative (Human Proteome Organization) www.hupo.org/research/hai 